\title{
Wavepacket models for supersonic jet noise
}

\author{
Aniruddha Sinha ${ }^{1} \dagger$, Daniel Rodríguez ${ }^{2}$, Guillaume A. Brès ${ }^{3}$ \\ and Tim Colonius ${ }^{1}$ \\ ${ }^{1}$ Engineering and Applied Sciences, California Institute of Technology, Pasadena, CA 91125, USA \\ ${ }^{2}$ School of Aeronautics, Universidad Politécnica de Madrid, E-28040 Madrid, Spain \\ ${ }^{3}$ Cascade Technologies Inc., Palo Alto, CA 94303, USA
}

(Received 3 September 2013; revised 30 October 2013; accepted 6 December 2013; first published online 21 February 2014)

Gudmundsson and Colonius (J. Fluid Mech., vol. 689, 2011, pp. 97-128) have recently shown that the average evolution of low-frequency, low-azimuthal modal large-scale structures in the near field of subsonic jets are remarkably well predicted as linear instability waves of the turbulent mean flow using parabolized stability equations. In this work, we extend this modelling technique to an isothermal and a moderately heated Mach 1.5 jet for which the mean flow fields are obtained from a high-fidelity large-eddy simulation database. The latter affords a rigourous and extensive validation of the model, which had only been pursued earlier with more limited experimental data. A filter based on proper orthogonal decomposition is applied to the data to extract the most energetic coherent components. These components display a distinct wavepacket character, and agree fairly well with the parabolized stability equations model predictions in terms of near-field pressure and flow velocity. We next apply a Kirchhoff surface acoustic propagation technique to the near-field pressure model and obtain an encouraging match for far-field noise levels in the peak aft direction. The results suggest that linear wavepackets in the turbulence are responsible for the loudest portion of the supersonic jet acoustic field.

Key words: absolute/convective instability, aeroacoustics, jet noise

\section{Introduction}

High exhaust noise levels associated with supersonic jets of military aircraft pose health problems for aircraft carrier personnel and community noise issues. Reduction of supersonic jet noise is thus a significant research challenge for future aircraft. Increases in bypass ratio, which have reduced subsonic jet noise in the commercial sector, are inappropriate for supersonic designs that favour small jet diameters, low drag and weight, and high specific thrust, all of which result in very high exhaust velocities. Current programs directed at jet noise reduction are demonstrating benefits of several decibels using passive and active control methods to increase jet mixing and break up shock cells in the over-expanded flow (e.g. Alkislar, Krothapalli \& Butler 2007; Henderson 2010; Samimy et al. 2012).

$\dagger$ Email address for correspondence: sinha.aniruddha@gmail.com 
Noise reduction through novel nozzle designs and active/passive flow control requires a large number of cost-function (far-field noise) evaluations in the process of optimization and control strategy identification. In the past, these efforts relied primarily on laboratory and full-scale experiments using a build-and-test approach. Although significant progress has been made in high-performance computing towards massively parallel simulation capabilities, such large parametric design studies also remain a prohibitively expensive task for high-fidelity computations with current resources. Thus, reduced-order (computationally efficient but approximate) models of the essential dynamics are invaluable for this effort in the near term. Recently, Kerhervé et al. (2012) reported a strategy for reduced-order modelling of unforced jets to predict their noise signatures. The authors used far-field noise data to educe the acoustically important parts of the shear layer fluctuations, followed by a system identification approach to model their dynamics. Gudmundsson \& Colonius (2011), on the other hand, demonstrated the possibility of using a more fundamental approach based on instability wavepackets to reduced-order modelling of jet noise, and this technique is extended here.

Mollo-Christensen (1963, 1967) first established the wavepacket behaviour of the near acoustic field of natural jets. Around the same time, coherent structures, reminiscent of linear instability waves, were being identified in forced jets and planar shear layers (Crow \& Champagne 1971; Brown \& Roshko 1974). Advecting large-scale structures in the physical flow domain correspond to low-frequency and low-azimuthal wavenumber wavepackets in the Fourier domain. The wavepackets in the jet shear layer constitute a relatively small fraction of the total disturbance energy (Cavalieri et al. 2013). However, they are acoustically significant owing to their high space-time coherence compared with the integral scales of turbulence (Tam \& Burton 1984; Jordan \& Colonius 2013), and our present results substantiate this view.

The discovery of wavepackets in the near field of turbulent jets was immediately followed by a sustained effort to use linear stability theory to describe their observed features. Early modelling efforts were directed exclusively at harmonically forced jets, owing to the ease of educing wavepackets therein (e.g. Mattingly \& Chang 1974; Crighton \& Gaster 1976; Michalke 1984; Tam \& Morris 1985). These comparisons with experimental data generally showed only qualitative agreement of the most amplified frequencies, wavelengths and growth/decay rates. As pointed out by Crighton \& Gaster (1976), nonlinearities might have been introduced by forcing at non-trivial amplitudes, further complicating the comparisons. The modelling of wavepackets in unforced jets was also attempted (e.g. Tam \& Chen 1994; Balakumar 1998; Yen \& Messersmith 1998; Piot et al. 2006), but this was hampered owing, in hindsight, to a lack of detailed spatiotemporal data. Furthermore, none of these studies showed how a linear instability wave could be consistent with the fluctuations of a real turbulent jet. That is, the precise choice of the base flow and the rationale for linearity remained open questions. The work of Mankbadi \& Liu (1984) was one of the few to account for weak nonlinearities, although the reliance on a number of empirical parameters limited the predictive capability of their model.

Tam (1971) hypothesized that the frequency of the spectral peak of supersonic jet mixing noise, as well as the polar directivity of this frequency, are associated with direct Mach wave radiation by instability waves. This mechanism is analogous to noise generation by supersonic flow over a wavy wall, and it requires the waves to have supersonic phase speed. Tam \& Burton (1984) pointed out that the broadband acoustic spectrum and broad directivity pattern of jet noise might be explained by modifying the wavy wall mechanism to account for the growth and decay of the 
instability waves. These latter aspects allow even subsonic jets to radiate noise. There were many attempts to validate this theory that were viewed as successful (e.g. Troutt \& McLaughlin 1982; Tam \& Hu 1989; Tam \& Chen 1994; Balakumar 1998; Yen \& Messersmith 1999; Lin et al. 2004; Piot et al. 2006), and the earlier efforts were reviewed by Tam (1991, 1995). However, the comparisons were actually based on limited measurements, and the lack of consistent identification of both the near and far sound fields prevented a more rigourous test of the theory. In fact, in an early attempt at quantifying the theory using a direct numerical simulation (DNS) database of a round jet at a Reynolds number based on the nozzle exit diameter $(R e)$ of 2000, Mohseni, Colonius \& Freund (2002) judged the linear theory to be inadequate, especially for modelling off-peak frequencies. In the DNS of an $R e=3600$ jet performed by Suponitsky, Sandham \& Morfey (2010), nonlinear interactions were also found to be an important mechanism of sound generation. However, we now have to question the relevance of these conclusions for turbulent jets, as they were based on initially laminar or transitional jets. In fact, the subsequent analytical work of Goldstein \& Leib (2005) lends support to the earlier (and present) view of the importance of linear instability waves of the turbulent mean flow field in determining the dominant aft angle sound radiation.

Recently, Suzuki \& Colonius (2006), Gudmundsson \& Colonius (2011) and Cavalieri et al. (2013) definitively demonstrated that wavepackets do exist in the near field of subsonic natural turbulent jets at high Reynolds numbers. The key differentiators from earlier studies were the availability of extensive data, and the application of an appropriate spatiotemporal filter to educe the wavepackets from them, namely proper orthogonal decomposition (POD) in the frequency domain. Moreover, linear stability theory was also found to be very satisfactory in modelling the average wavepackets thus observed when the measured mean velocity field was used as the base flow. The first of these studies used the classical parallel flow stability theory. The subsequent models were constructed with parabolized stability equations (PSE) that accounts for the slow divergence of the mean flow, and significantly improved agreement was found with experiments. These recent advances in experiments and theory for subsonic jets were reviewed by Jordan \& Colonius (2013).

Regarding the prediction of actual sound emission, the supersonic case is more straightforward than the subsonic. In the latter, although there is little doubt that the wavepackets play a role in the peak frequency emission, their intermittency, which cannot be predicted by the theory, appears to have a substantial amplifying effect over the entire spectrum (Cavalieri et al. 2011). Apart from the issue of intermittency, the consideration of the supersonic jet affords the direct acoustic modelling with PSE in another manner. Cheung \& Lele (2009) demonstrated that PSE successfully predicts the acoustic field in a laminar supersonic mixing layer, but fails in a subsonic case: a hybrid PSE-acoustic analogy approach was used to circumvent the latter problem. This behaviour is explicated in our subsequent discussion.

We revisit the supersonic jet noise problem here, and test whether we can apply similar modelling and eduction techniques as Gudmundsson \& Colonius (2011) to accurately account for both the near-field wavepackets as well as their radiated sound, all in a linear framework. The present investigation is materially facilitated by the availability of a high-fidelity large-eddy simulation (LES) database consisting of two ideally expanded convectively supersonic round jets: one isothermal and the other moderately heated (Brès et al. 2012). This database, in fact, allows us to progress farther than the earlier studies in that we can use the full volumetric data in a more rigourous and detailed validation of the theory. 
The agreement demonstrated here for both the near and far fields suggests that Tam's mechanism for sound generation is correct, even quantitatively, in a real turbulent flow field. We also learn that, unlike in subsonic jets, the intermittency of wavepackets plays a minor role in supersonic jet noise. One question that this investigation omits addressing is the reason behind the wavepackets' linear behaviour. Gudmundsson \& Colonius (2011) advanced a possible explanation based on the theory of marginal stability (Malkus 1956), but this remains a conjecture at this point.

\section{Theory}

\subsection{Instability wave models using PSE}

Instability waves in the turbulent jet are modelled with linear parabolized stability equations following the description in Gudmundsson \& Colonius (2011); a brief review of the procedure appears below.

The usual compressible formulation is used to non-dimensionalize flow quantities. Linear dimensions are normalized by the nozzle exit diameter $D$, velocities by the ambient speed of sound $c_{\infty}$, density by the ambient density $\rho_{\infty}$ and pressure by $\rho_{\infty} c_{\infty}^{2}$. Time is normalized by $D / c_{\infty}$. However, for the purposes of reporting, frequency is normalized by $U_{j} / D$ to the more common Strouhal number $S t$, where $U_{j}$ is the nozzle exit velocity. The acoustic Mach number of the jet is $M_{a}=U_{j} / c_{\infty}$. The Reynolds number is $\operatorname{Re}=\rho_{j} U_{j} D / \mu_{j}$, with $\rho_{j}$ and $\mu_{j}$ being respectively the density and viscosity at the nozzle exit. In the instability wave model, the temperature dependence of viscosity is ignored due to the small temperature ratio of the jets considered. Moreover, the Prandtl number $\mathrm{Pr}$ is fixed at 0.7 for air. Finally, the equation of state assumes an ideal gas with constant ratio of specific heats $\gamma=1.4$.

The jet flow field is described in cylindrical coordinates by $\boldsymbol{q}=\left(u_{x}, u_{r}, u_{\theta}, p, \zeta\right)^{\mathrm{T}}$, which denote the axial, radial and azimuthal components of velocity, the pressure and specific volume (the reciprocal of density, $\rho$ ), respectively. The instability waves are modelled as perturbations $\boldsymbol{q}^{\prime}$ of the time-averaged and azimuth-averaged turbulent flow field $\overline{\boldsymbol{q}}$, i.e. $\boldsymbol{q}(x, r, \theta, t)=\overline{\boldsymbol{q}}(x, r)+\boldsymbol{q}^{\prime}(x, r, \theta, t)$. The time-stationarity and azimuthal homogeneity of round jets afford Fourier decompositions of $\boldsymbol{q}^{\prime}$ in the corresponding dimensions:

$$
\boldsymbol{q}^{\prime}(x, r, \theta, t)=\sum_{m, \omega} \hat{\boldsymbol{q}}_{m, \omega}(x, r) \exp \{\mathrm{i}(m \theta-\omega t)\}
$$

Here, $\omega=2 \pi S t M_{a}$ is the angular frequency, $m$ is the azimuthal wavenumber and $\hat{\boldsymbol{q}}$ is the spatial Fourier coefficient. Here and elsewhere, the subscripts of $m$ and $\omega$ will be omitted from the notation unless required for clarity. The PSE model assumes that $\hat{\boldsymbol{q}}$ can be decomposed into a rapidly varying wave-like component modulated by a function with axial variations on the order of the base flow:

$$
\hat{\boldsymbol{q}}_{m, \omega}(x, r)=B_{m, \omega} \tilde{\boldsymbol{q}}_{m, \omega}(x, r) \chi_{m, \omega}(x), \quad \chi_{m, \omega}(x)=\exp \left\{\mathrm{i} \int_{x_{0}}^{x} \alpha_{m, \omega}(\xi) \mathrm{d} \xi\right\} .
$$

Here, $\tilde{\boldsymbol{q}}$ is a shape function and $\alpha$ is a complex axial wavenumber, both assumed to have mild axial variation. Finally, $B$ is a complex scalar that sets the absolute amplitude and phase of the PSE solution. The decomposition in (2.2) is ambiguous since the axial variation can be subsumed in either $\tilde{\boldsymbol{q}}$ or $\alpha$. Herbert (1997) prescribed the following normalization constraint

$$
\int_{0}^{\infty} \sum_{j \in\{x, r, \theta\}} \tilde{u}_{j}^{\dagger} \frac{\partial \tilde{u}_{j}}{\partial x} r \mathrm{~d} r=0
$$


where $(\cdot)^{\dagger}$ denotes the complex conjugate. This aims to remove any exponential dependence on $x$ from the shape function $\tilde{\boldsymbol{q}}$.

Introducing the decomposition (2.1), (2.2) into the compressible Navier-Stokes, continuity and energy equations, and projecting them on to the retained Fourier basis, yields the following system of equations for each retained Fourier mode pair

$$
\left(A+B \frac{\mathrm{d} \alpha_{m, \omega}}{\mathrm{d} x}+C \frac{\partial}{\partial x}+D \frac{\partial}{\partial r}+E \frac{\partial^{2}}{\partial r^{2}}+F \frac{\partial^{2}}{\partial x \partial r}\right) \tilde{\boldsymbol{q}}_{m, \omega}=\frac{\tilde{R}_{m, \omega}}{B_{m, \omega} \chi_{m, \omega}} .
$$

The linear operators $A$ through $F$ are functions of $\overline{\boldsymbol{q}}, \omega, m$ and $\alpha$; expressions for them can be obtained from the linearized governing equations presented in appendix A. All nonlinear terms are gathered in $\tilde{R}$. Following on the success of linear PSE in modelling subsonic jet wavepackets, the nonlinear terms are neglected in the present work. This renders the equations decoupled and homogenous, so that they neither depend on nor predict the absolute amplitude $B$. Under the assumption of slow $x$ variations of $\tilde{\boldsymbol{q}}, \partial^{2} \tilde{\boldsymbol{q}} / \partial x^{2}$ is omitted in (2.4) (although the other terms in $\partial^{2} \hat{\boldsymbol{q}} / \partial x^{2}$ are retained), which renders the equations approximately parabolic for convectively unstable flows such as the jets under consideration (Li \& Malik 1997).

Equation (2.4) is closed in the radial direction at $r=15$ by characteristic boundary conditions following Thompson (1987), and the pole condition at the centreline is implemented as per Mohseni \& Colonius (2000). The radial grid is clustered on the jet lip line (with a minimum spacing of 0.004D) where the shear is largest following Freund (1997), and fourth-order central difference is used to discretize the radial derivative operators. First-order implicit Euler differences are used to approximate the axial derivatives, and this results in a system of equations to solve for the shape functions at each axial position, given a guess for $\alpha$. The latter is solved for iteratively to satisfy (2.3), as shown by Day, Mansour \& Reynolds (2001).

For stable downstream march of the solution, Li \& Malik (1997) specify the following lower bound on the axial step size

$$
\Delta x \geqslant \frac{1}{\left|\operatorname{Re}\left\{\alpha_{m, \omega}(x)\right\}\right|} .
$$

Marching with the minimum allowable $\Delta x$ has been found to be necessary for proper resolution of the acoustic field, so that each Fourier mode is solved on its own $x$ grid as dictated by (2.5). To facilitate post-processing, both $\tilde{\boldsymbol{q}}$ and $\alpha$ solutions are interpolated linearly on to a uniform $x$ grid, their slow $x$ variation rendering higherorder interpolation superfluous.

An upstream condition (akin to the initial condition for time marching) is required to begin the axial march at $x=x_{0}$. This is obtained by solving the classical parallel-flow linear stability problem based on the mean flow profile close to the nozzle exit, and extracting the Kelvin-Helmholtz $(\mathrm{K}-\mathrm{H})$ mode. Even though supersonic jets support other unstable modes (Tam \& $\mathrm{Hu} 1989$ ), the $\mathrm{K}-\mathrm{H}$ mode undergoes the largest amplification, and thus governs the wavepacket dynamics (Rodríguez et al. 2013).

\subsection{Kirchhoff surface formulation for acoustic field}

The acoustic field of the PSE solution is desired. The simple Kirchhoff surface (KS) formulation described below has been used in continuing the acoustic solution of jets from direct numerical simulations (e.g. Freund 2001), as well as from PSE models (Balakumar 1998; Lin et al. 2004). Following Lighthill (1952), one starts by formally recasting PSE into the forced Helmholtz equation for pressure fluctuations in 
the $m-\omega$ Fourier domain:

$$
\left\{\left(1-M_{c o}^{2}\right) \frac{\partial^{2}}{\partial x^{2}}+2 \mathrm{i} \omega M_{c o} \frac{\partial}{\partial x}+\frac{1}{r} \frac{\partial}{\partial r}\left(r \frac{\partial}{\partial r}\right)+\left(\omega^{2}-\frac{m^{2}}{r^{2}}\right)\right\} \hat{p}_{m, \omega}(x, r)=\hat{\mathscr{S}}_{m, \omega}(x, r) .
$$

Here, the source term $\hat{\mathscr{S}}$ aggregates all of the terms in the PSE that are required to balance the left-hand side (LHS) of (2.6). For generality that would be useful subsequently, a uniform axial 'coflow' around the jet extending to the far-field observer is assumed, with Mach number $M_{c o}$ referred to the ambient speed of sound (Morse \& Ingard 1968).

Since the acoustic source in practical jet flow fields has compact support in the axial domain, the following axial Fourier transform is well defined:

$$
\check{p}_{m, \omega}(k, r):=\int_{-\infty}^{\infty} \hat{p}_{m, \omega}(x, r) \mathrm{e}^{-\mathrm{i} k x} \mathrm{~d} x, \quad \hat{p}_{m, \omega}(x, r)=\frac{1}{2 \pi} \int_{-K}^{K} \check{p}_{m, \omega}(k, r) \mathrm{e}^{\mathrm{i} k x} \mathrm{~d} k,
$$

where $K$ is the integration bound on the axial wavenumber $k$ set by the practical resolution in $x$. Applying an analogous axial Fourier transform to the source term $\hat{\mathscr{S}}$, the acoustic analogy in (2.6) reduces to

$$
\left[\frac{\partial^{2}}{\partial r^{2}}+\frac{1}{r} \frac{\partial}{\partial r}+\left\{\left(\omega+k M_{c o}\right)^{2}-k^{2}-\frac{m^{2}}{r^{2}}\right\}\right] \check{p}_{m, \omega}(k, r)=\check{\mathscr{S}}_{m, \omega}(k, r) .
$$

The acoustic source $\check{\mathscr{S}}$ is compact in the radial direction too, as we will demonstrate. Thus, equation (2.8) is essentially homogenous beyond a certain radius, say $r_{K S}$, that is within the physical domain of the PSE solution but outside the jet flow. The linear acoustic field for $r>r_{K S}$ can be determined by treating the homogenous version of (2.8) as a boundary value problem, with $\breve{p}_{m, \omega}(k, r)$ specified on the cylindrical shell $r=r_{K S}$. Applying a radiation condition at $r \rightarrow \infty$ restricts the solution for positive $\omega$ to be

$$
\check{p}_{m, \omega}(k, r)=\check{p}_{m, \omega}\left(k, r_{K S}\right) H_{m}^{(1)}(\Lambda r) / H_{m}^{(1)}\left(\Lambda r_{K S}\right), \quad \Lambda=\sqrt{\left(\omega+k M_{c o}\right)^{2}-k^{2}},
$$

where $H_{m}^{(1)}$ is the $m$ th-order Hankel functions of the first kind. The inverse Fourier transform specified in (2.7) then yields the desired acoustic field $\hat{p}_{m, \omega}(x, r)$ associated with a given PSE mode $\hat{\boldsymbol{q}}_{m, \omega}(x, r)$ for $r>r_{K S}$. In this study, the evaluation of (2.9) considers the radiating $k$ modes solely; these are given by $-\omega /\left(1+M_{c o}\right)<k<\omega /\left(1-M_{c o}\right)$.

\section{LES database and its processing}

\subsection{Description of database}

An LES database of two ideally expanded supersonic round jets is used in the present work. The flow conditions (listed in table 1) match the experimental conditions in the United Technologies Research Center (UTRC) anechoic open-jet facility (Schlinker et al. 2009). Note that the nozzle exit Mach number $M_{j}=U_{j} / c_{j}$ is 1.5 for both jets. For both cases, the experiments have a wind tunnel coflow of Mach number $M_{c o}=$ 0.1 extending to $r=10$ in the radial direction. To try to replicate the UTRC test conditions, the simulations include the same coflow across the whole computational domain. 


\begin{tabular}{|c|c|c|c|c|c|c|}
\hline Case & Description & $M_{j}$ & $T_{j} / T_{\infty}$ & $M_{a}$ & $\operatorname{Re}$ & Simulation duration \\
\hline B118 & Isothermal ideally expanded & 1.5 & 1.0 & 1.5 & 300000 & 215 \\
\hline B122 & Heated ideally expanded & 1.5 & 1.74 & 1.98 & 155000 & 112 \\
\hline
\end{tabular}

TABLE 1. Jet operating conditions.

The simulations were performed using the flow solver 'Charles' developed at Cascade Technologies (Brès et al. 2012). The spatially filtered compressible NavierStokes equations are solved on an unstructured grid using a control-volume-based finite volume method. The flux at each control-volume face is computed using a blend of a non-dissipative central flux and a dissipative upwind flux. The blending parameter is precomputed based on the grid and the differencing operators using a heuristic algorithm to minimize numerical dissipation while ensuring stability. The Vreman subgrid-scale model (Vreman 2004; You \& Moin 2007) is used with constant coefficients $\left(c=0.07, P r_{t}=0.9\right)$ to account for the physical effects of the unresolved turbulence on the resolved flow. The shock-capturing scheme available in the flow solver (Brès et al. 2012) is inactive since only residual and weak shocks are present at the nominally ideally expanded conditions simulated here.

The round converging-diverging nozzle geometry from the UTRC experiments (designed using the method of characteristics) is included in the computational domain, with adiabatic no-slip wall boundary conditions applied on the entire nozzle surface. A constant plug flow is applied to the inlet of the nozzle such that the desired conditions are attained at its exit plane. The flow issued from the nozzle is laminar; the corresponding condition has not been measured in the experiments. The momentum thickness of the boundary layer at the nozzle exit is approximately $0.0017 D$ and $0.0022 D$ in the B118 and B122 cases, respectively. The use of such thin laminar boundary layers leads to rapid transition to turbulence near the nozzle exit (approximately one nozzle diameter downstream in the present cases) while affording coarser resolution inside the nozzle.

The computational domain extends to $45 D$ in the axial direction; in the radial direction, the domain extent grows from $12 D$ at the inflow plane to $20 D$ at the downstream outflow boundary. The typical aeroacoustic treatments are applied near the outlets of the computational domain to avoid spurious reflections (Brès et al. 2012). The mesh consists of a fully unstructured core which transitions to a purely axisymmetric grid with 160 points in the azimuthal direction and limited stretching within the Ffowcs Williams-Hawkings (FW-H) surface (described in $\S 6$ ), followed by further stretching towards the domain boundaries. Both the simulations in this work were performed on a mesh containing approximately 42 million control volumes. The non-dimensional simulation time durations (after initial transients) are reported in table 1. These durations can be considered as long time samples of high-fidelity LES, thus ensuring the statistical convergence of the stationary quantities and also a reasonable convergence of the low-frequency noise spectra. For both cases, the data were saved at intervals of 0.02 , such that the LES provide reliable results at high frequency for $S t$ up to nearly 10.

Good agreement was found in extensive comparisons with the experiments at UTRC considering flow field statistics and near and far-field pressure spectra (Brès et al. 2012). Time-averaged axial velocity fields for the two simulated jets are shown in figure 1. The two LES mean fields are used without further smoothing 

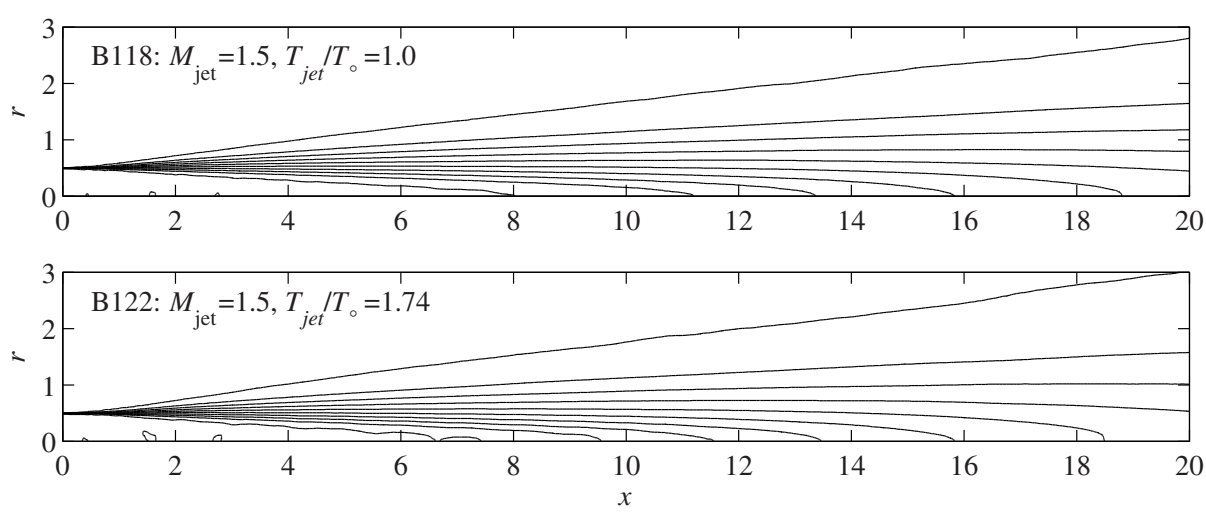

FIgURE 1. Contours of axial velocity $\bar{U}_{x} / U_{j}$ for the two jets under consideration. Contours are in equal increments from 0.1 to 0.99 .
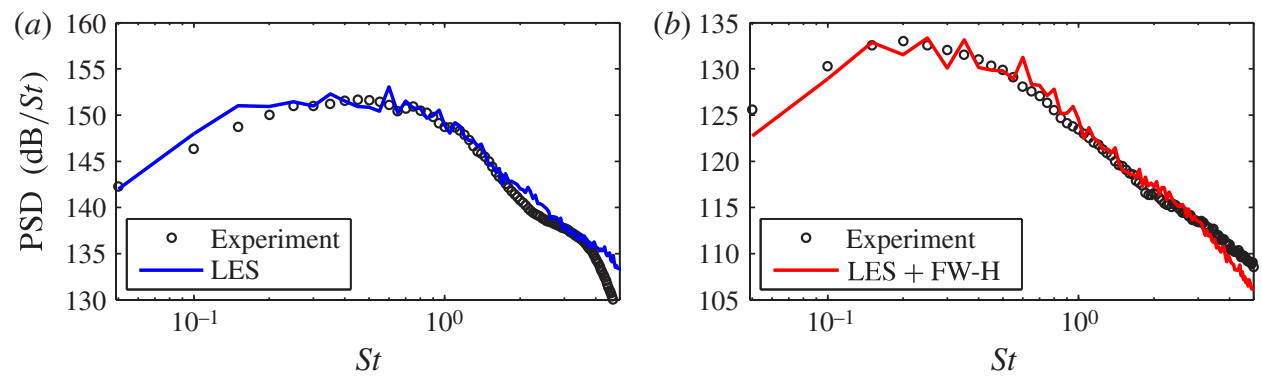

Figure 2. (Colour online) Comparison of sound spectra in the isothermal (B118) jet in $(a)$ the near field at $x=5, r=1.5$ and $(b)$ the far field at a polar angle of $145^{\circ}$ (measured from the upstream jet axis) and polar radius of 70.2 nozzle diameters (with origin at the centre of the nozzle exit plane).

as the base flows for the respective PSE models. Figure 2 presents instances of the fidelity of the near-field pressure and far-field acoustics. The former corresponds to the direct prediction in the LES, whereas the latter is computed using the FW-H formulation. The predicted spectra are bin-averaged with $\Delta S t_{\text {bin }}=0.05$. This choice of bin-averaging, along with the short time signal compared to experiments, are the main reasons for the relative lack of smoothness of the spectra at the low frequencies. The data-processing techniques are briefly described in $\S 6$, and detailed by Brès et al. (2012).

The database of time-varying flow fields is large, in the tens of terabytes for each case. To recover a manageable dataset, the flow variables are under-sampled in spatial resolution. In particular, a linear interpolation is performed onto a cylindrical grid. The axial and azimuthal grids are uniform with 321 and 24 points, respectively; the axial grid extends between $x=0$ and 20. The non-uniform radial grid has 176 points between $r=0$ and 5 with clustering on the lip line to yield a minimum spacing of $0.0076 D$. 


\subsection{Wavepacket eduction}

The PSE model is intended to represent an average wavepacket that is, by definition, coherent over the entire flow domain (albeit with possibly trivial amplitude in null regions). On the other hand, LES resolves motions over a broad range of spatial and temporal scales. Thus, for the purposes of validating the PSE model with the LES database, appropriate statistical techniques must be applied on the latter to educe the signatures of flow structures that correlate over significant spatial regions. In previous work considering subsonic jets, POD was applied towards this end, either to the pressure acquired on a phased microphone array in the near field (Gudmundsson \& Colonius 2011), or to the velocity fluctuations measured on cross-sections using time-resolved particle image velocimetry (Cavalieri et al. 2013). The LES database used in the present validation permits great flexibility in the computation of POD modes, since all flow variables are available on the entire relevant flow domain.

Uppercase symbols are used to denote the empirical jet flow field; cf. the lowercase symbols used to denote the modelled flow field. Thus, the empirical flow field vector is $\boldsymbol{Q}=\left(U_{x}, U_{r}, U_{\theta}, P, \Xi\right)^{\mathrm{T}}$, with the components having meaning analogous to those of $\boldsymbol{q}$. Moreover, as for $\boldsymbol{q}$, the empirical flow field $\boldsymbol{Q}$ is subjected to Reynolds decomposition into the mean and fluctuations, as well as Fourier decomposition into frequency and azimuthal modes.

Prior to the temporal Fourier transform, the empirical time record is divided into $J$ segments with Hann windowing (similar to the Welch spectrogram method), and the individual segments are considered to be independent realizations of the flow. The time segments have a $75 \%$ overlap and correspond to a frequency bin size of $\Delta S t=0.025$. The available time records (see table 1) result in $J=29$ and 19 segments for the B118 and B122 cases, respectively. The Fourier transformed flow field in the $j$ th segment is denoted by $\hat{\boldsymbol{Q}}_{m, \omega}^{[j]}(x, r)$.

A systematic investigation of different inner products in the computation of POD modes of a turbulent jet was conducted by Freund \& Colonius (2009), showing that the resulting decomposition materially depends on the physical variables retained. The near-field pressure of turbulent jets displays the wavepacket character most clearly, which is attributable to the wavenumber-filtering behaviour of the pressure field (Suzuki \& Colonius 2006; Gudmundsson \& Colonius 2011; Jordan \& Colonius 2013). It has also been established in (2.9) that the accurate modelling of the near-field pressure is sufficient for obtaining the correct far field, which is the ultimate goal of this research. The velocity field, on the other hand, prominently displays the effect of vorticity and entropy modes that are not modelled in this work. Thus, the inner product between two fields $\hat{\boldsymbol{Q}}^{[1]}$ and $\hat{\boldsymbol{Q}}^{[2]}$ is defined here as

$$
\left\langle\hat{\boldsymbol{Q}}^{[1]}, \hat{\boldsymbol{Q}}^{[2]}\right\rangle_{P}:=\int_{x=0}^{20} \int_{r=0}^{5}\left\{\hat{P}^{[2]}(x, r)\right\}^{\dagger} \hat{P}^{[1]}(x, r) r \mathrm{~d} r \mathrm{~d} x .
$$

The domain of integration is determined by the availability of empirical data, as described in $\S 3.1$.

The frequency-domain variant of the snapshot POD method of Sirovich (1987) is employed here. In this technique, the POD spatial eigenfunction $\hat{\boldsymbol{\Phi}}_{m, \omega}(x, r)$ is expressed as a linear combination of the available set of realizations:

$$
\hat{\boldsymbol{\Phi}}_{m, \omega}(x, r)=\sum_{j=1}^{J} \beta_{m, \omega}^{[j]} \hat{\boldsymbol{Q}}_{m, \omega}^{[j]}(x, r) .
$$




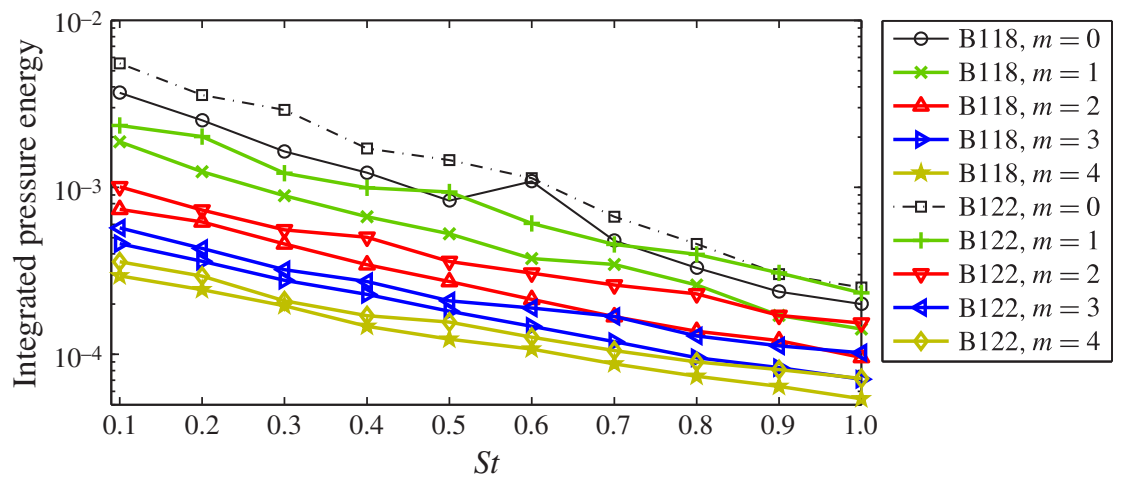

Figure 3. (Colour online) Pressure energy integrated over the domain $0 \leqslant x \leqslant 20,0 \leqslant r \leqslant$ 5 in various $m-S t$ Fourier modes of the LES data of the isothermal (B118) and heated (B122) jets.

Then, the weight coefficients $\beta$ are obtained as eigenvectors from the following eigenvalue problem

$$
\sum_{j=1}^{J} \mathscr{T}_{m, \omega}^{j i} \beta_{m, \omega}^{[j]}=\lambda_{m, \omega} \beta_{m, \omega}^{[i]}, \quad \forall i \in[1, J], \quad \mathscr{T}_{m, \omega}^{i j}:=\frac{1}{J}\left\langle\hat{\boldsymbol{Q}}_{m, \omega}^{[i]}, \hat{\boldsymbol{Q}}_{m, \omega}^{[j]}\right\rangle_{P}
$$

Note that, although the decomposition is solely determined by the pressure field, the other components of the flow field that are correlated with the POD modes of pressure are also retrieved through (3.2).

The kernel $\mathscr{T}$ is Hermitian so that the eigenvalues $\lambda^{(n)}$ (indexed by $n$ ) are nonnegative; they are ordered such that $\lambda^{(n)} \geqslant \lambda^{(n+1)}$. A faster rate of decay indicates higher coherence in the data, since the POD eigenfunctions $\hat{\boldsymbol{\Phi}}^{(n)}$ are orthogonal with respect to the inner product in (3.1). To render the amplitudes of the POD modes directly comparable with the fluctuation energy of the flow, the normalization of the eigenfunctions is such that $\left\|\hat{\boldsymbol{\Phi}}^{(n)}\right\|_{P}=\sqrt{\lambda^{(n)}}$, the norm being induced from (3.1).

\section{Comparisons of near-field pressure}

In this section, the near-field pressure predicted by the linear PSE model will be compared with the POD modes of pressure computed from the LES database. As a preliminary step, figure 3 presents the integrated pressure energy; this is defined as the ensemble-averaged value of the square of the norm induced from (3.1). Here and hereafter, the statistics of the $+m$ and $-m$ modes are averaged before presentation. The integrated energy decreases very rapidly with increasing azimuthal mode, $m=$ 0 being an order-of-magnitude more energetic than $m=2$ at low frequencies. The pressure fluctuations at higher frequencies are confined over smaller $x$-regions (see later), which accounts for the rapid decrease in integrated energy with $S t$. The trends are identical for the isothermal and heated jets, with the latter displaying more energy. This graph justifies the subsequent focus on the first three azimuthal modes, and to $S t \leqslant 1$; these Fourier modes also predominate the far-field acoustics. The $S t=0.6, m=$ 0 mode of the B118 case appears to be anomalously energetic. Possible reasons for this may be the weak shocks present in the flow, or the effect of vortex pairing in the 


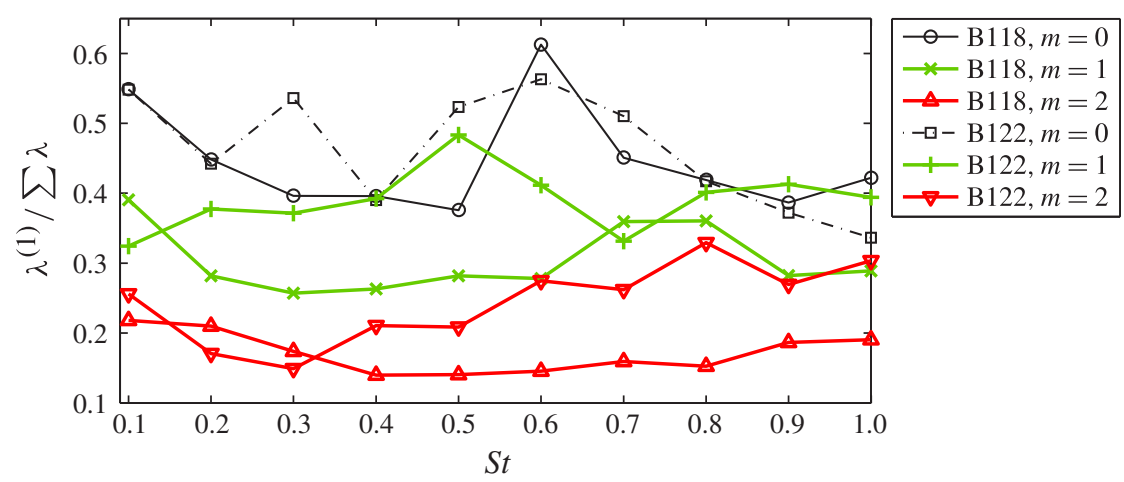

FIGURE 4. (Colour online) Fraction of pressure fluctuations in the first POD modes of various $m-S t$ Fourier modes for the LES data of the isothermal (B118) and heated (B122) jets.

initial transitional zone. The validation of the present linear model is not affected by this anomaly.

The fraction of total pressure energy represented by the first POD modes of various relevant Fourier modes is shown in figure 4. The first POD mode captures approximately $50 \%$ of the fluctuation energy for $m=0$, and $\sim 35 \%$ for $m=1$, across the range of St depicted. Recalling the large $x-r$ domain of the POD that greatly exceeds the integral length scales of the flow, this attests to very significant coherence for all of the modes depicted. The $m=2$ mode is less coherent. The results for the isothermal and heated jets are similar, indicating that heating does not have a marked effect on coherence.

Since the absolute amplitude of wavepackets is indeterminate in linear PSE, the most relevant metric for comparison is the 'alignment' of the PSE prediction with the $n$th POD mode for a particular Fourier mode. This alignment is calculated as follows

$$
\left[\mathscr{A}_{P}\right]_{m, \omega}^{(n)}:=\frac{\left|\left\langle\hat{\boldsymbol{q}}_{m, \omega}, \hat{\boldsymbol{\Phi}}_{m, \omega}^{(n)}\right\rangle_{P}\right|}{\left\|\hat{\boldsymbol{q}}_{m, \omega}\right\|_{P}\left\|\hat{\boldsymbol{\Phi}}_{m, \omega}^{(n)}\right\|_{P}} .
$$

Since the POD modes form an orthogonal basis, the above definition implies that $0 \leqslant\left[\mathscr{A}_{P}\right]^{(n)} \leqslant 1$ and $\sum_{n}\left(\left[\mathscr{A}_{P}\right]^{(n)}\right)^{2}=1$. A value close to unity for the first POD mode indicates that the PSE solution is structurally equivalent to the most coherent wavepacket found in the flow.

Figure 5 presents the alignment metric of (4.1) to compare the PSE solution for the isothermal supersonic jet with the first two POD modes of the LES data. Overall, the PSE model predictions demonstrate good agreement with the most energetic wavepackets (the first POD mode) extracted from the database for $m=0$ and 1 . Modelling the low-frequency axisymmetric modes with PSE has proven challenging for subsonic jets previously (Gudmundsson \& Colonius 2011), and they continue to be so in the present supersonic case, with $S t \leqslant 0.2, m=0$ modes displaying poorer agreement; we will revisit this point later. The agreement in $m=2$ is not as good; the probable reason for this is discussed subsequently.

The above analysis demonstrates that the PSE solution is most aligned with the first pressure POD mode of the LES data. The latter is then used to select the 


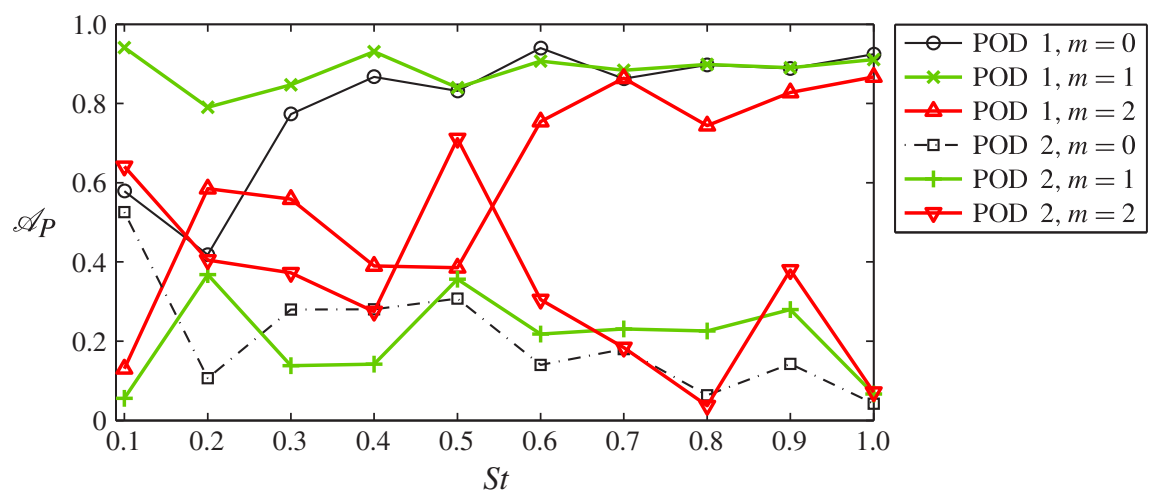

FIgURE 5. (Colour online) Alignment of the PSE solution with first and second POD modes of pressure for the isothermal (B118) jet.

complex amplitude factor of the PSE modes, $B_{m, \omega}$ (see (2.2)), using a least-squares fit (Gudmundsson \& Colonius 2011). This gives

$$
B_{m, \omega}=\frac{\left\langle\hat{\boldsymbol{\Phi}}_{m, \omega}^{(n)}, \chi_{m, \omega} \tilde{\boldsymbol{q}}_{m, \omega}\right\rangle_{P}}{\left\|\chi_{m, \omega} \tilde{\boldsymbol{q}}_{m, \omega}\right\|_{P}^{2}} .
$$

This scaling is consistently retained during the subsequent comparisons of the pressure and velocity components of the PSE solution, as well as the projected acoustic fields. Rodríguez et al. (2013) present an alternate method of determining the amplitudes of the PSE wavepackets based on an adjoint-based projection of the LES fluctuation data near the nozzle exit plane onto the parallel-flow linear stability modes used to initiate the PSE.

Figure 6 depicts visual comparisons of the linear PSE solutions with the first POD eigenfunctions for some of the Fourier modes under consideration. The real parts of the pressure components are plotted, and the contour levels are saturated to clarify the near acoustic fields. (The weak shock cells present in the numerical data appear amplified due to this plotting method.) This figure supplies an intuitive explanation for the alignment metrics presented in figure 5. Significant similarity is observed for all the Fourier modes except the lowest-frequency axisymmetric mode. In particular, the radiation patterns in the near pressure field that are most relevant for modelling the farfield noise are captured quite well by the PSE. The agreement is in all three aspects of the patterns, namely wavelength (equivalently, advection speed), axial location of the peak and polar angle of the directed radiation.

The relative success of PSE in predicting the average near acoustic field demonstrated above is linked to our choice of the supersonic jet as the test case. The PSE ansatz imposes a single complex wavenumber $\alpha$ at a given cross-section. However, the shape function $\tilde{\boldsymbol{q}}$ is allowed to distort locally within the constraint imposed by the normalization condition in (2.3). Thus, the modelled wavepacket $\hat{\boldsymbol{q}}$ may exhibit moderately different wavelengths in the hydrodynamic and acoustic regions. In the supersonic jets modelled here, the disparity of wavelengths in the two regions is not large (see figure 6, and also figure 8 appearing later), so that PSE is able to approximate both domains. The noise field associated with subsonic shear layers does not satisfy this condition: this explains the corresponding failure of PSE reported by Cheung \& Lele (2009). 

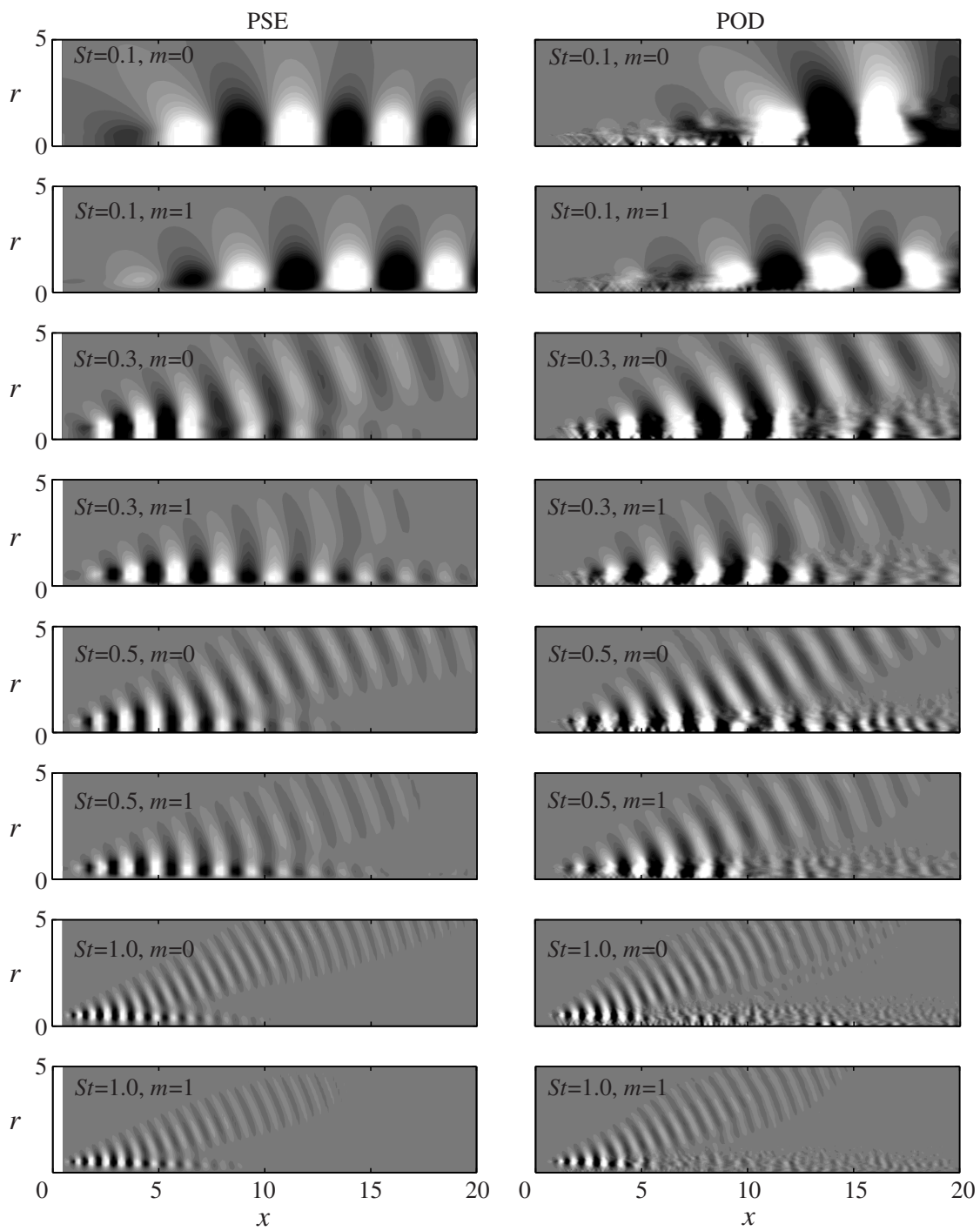

FIGURE 6. Comparison of pressure component of PSE solution with corresponding first POD modes for the isothermal (B118) jet. The real part of the modes are plotted with greyscale ranging within \pm 0.0034 .

Gudmundsson \& Colonius (2011) conjectured that a probable cause for the PSE modelling error in the low-St axisymmetric modes might be their violation of the slowly varying base flow assumption. Their wavelengths being on the order of the length of the potential core, these wavepackets are subject to relatively rapid variations of the base flow. However, subsequent studies with linearized Euler equations indicate that such discrepancies are encountered even when the mildly non-parallel assumption is relaxed (Baqui et al. 2013). Although not reported here, our recent preliminary investigation with nonlinear PSE supplies an alternative explanation for this discrepancy. We found that nonlinear effects on wavepacket evolution are strongest at low frequencies, but minimal for $S t \geqslant 0.3$. Low-frequency modes have lower growth rates, so that nonlinear coupling with other modes might 
have a relatively stronger influence. The nonlinear PSE studies being preliminary, this explanation should be considered as a conjecture at this point.

The agreement between PSE and POD in figure 6 degrades downstream within the jet core where the wavepackets' amplitudes have decayed. First, in these low-coherence regions of the flow, the POD modes may not be fully converged, given the limited data record available. More importantly, this disagreement does not signify a failure of PSE, which is intended to model the acoustically relevant dynamics of wavepackets in their energetic regime: the success in this aspect is encouraging in figure 6 , and further demonstrated in $\S 6$. Given these caveats, it is still conceivable that the inclusion of a richer set of modes (apart from the sole $\mathrm{K}-\mathrm{H}$ mode), even in a linear model, might have improved the agreement with POD. As the $\mathrm{K}-\mathrm{H}$ mode decays downstream, the coherent field may come to be dominated by vorticity and/or entropy modes that are triggered through the non-normality of the linearized Navier-Stokes operator. Another possible cause of the observed discrepancy may be the assumption of mild non-parallelism of the base flow: this is violated locally for low- to moderate-frequency wavepackets near the end of the potential core. Finally, the present results cannot discriminate the role of nonlinearities in the downstream region: low-frequency wavepackets that dominate thereat may be exciting the moderate- to high-frequency modes that are predicted to have decayed in the linear theory.

Investigation of the pressure contour plots for the $m=2$ modes (not presented here) reveals that the cause of their lower 'alignment' in figure 5 is related to the above. These modes, especially at the lower frequencies, have a weak near acoustic field, so that the modelling errors incurred in the downstream shear layer dominate the alignment metric.

The heated supersonic B122 jet is also modelled using linear PSE, and presentation of these results follows the preceding scheme. The eigenspectra for the B118 and B122 jets have been demonstrated to be quite similar in figure 4. Figure 7 shows that the alignment of the PSE solution with the first POD mode follows the trends found in the B118 case. Two differences are noted though: the misalignment is significant up to $S t=0.3$ in the $m=0$ mode (we have speculated on the cause of this discrepancy above), and the predictions of the $m=2$ modes in the mid range of frequencies are much improved (owing to their stronger near acoustic field).

Figure 8 presents visual comparisons of the PSE solutions with the first POD eigenfunctions. The general match between the two is encouraging. In particular, linear PSE correctly captures the increased polar angle of the peak radiation caused by the increased jet velocity, while accurately predicting the wavelength of the wavepackets.

The alignment metrics presented in figures 5 and 7 are also computed with POD modes obtained using only $80 \%$ of the available data. The results deviate by less than 0.05 , attesting to the robustness of the conclusions drawn thereof.

\section{Comparisons of the velocity field}

The axial and radial components of velocity predicted by PSE in the isothermal jet case are compared with those of the first POD modes for two representative Fourier modes in figure 9. We repeat that the POD is based on the pressure fluctuations alone, and the velocity fields presented are correlated with the pressure through the use of common weight coefficients for the frequential snapshots in (3.2). Furthermore, the complex scale factor applied to the PSE fields is retained from (4.2). 


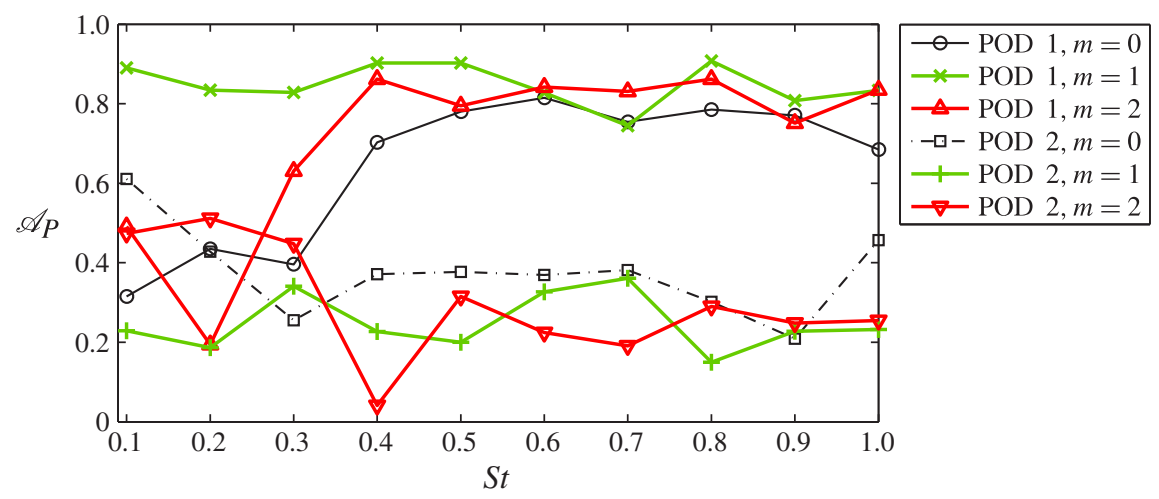

FIGURE 7. (Colour online) Alignment of PSE solution with first and second POD modes of pressure for the heated (B122) jet.

The PSE model predictions agree to a moderate degree with the empirical data, namely the wavelength and phase of wavepackets as well as their amplitude and shape, in the growing region. However, the degradation of fidelity is significant further downstream. Some possible sources of this discrepancy have been discussed in $\S 4$, although the disagreement is more severe for the velocity than for pressure. As the PSE mode adopts the correct near-field acoustic behaviour downstream (see figure 6), it exhibits the velocity oscillations associated with this linear eigenmode in the core since a single axial wavenumber is being imposed by the ansatz. Examples of such acoustic modes were presented by Rodríguez et al. (2013) for the jets under consideration here. PSE is unable to model the larger set of modes required to represent the full physics in this region. The predicted axial velocity field suffers from another inaccuracy: the radial gradient of the wavepacket at the outer edge of the shear layer is much sharper than that observed in the POD modes. This may be linked to the non-normality of the Navier-Stokes equations (see discussion in $\S 4)$. The strong, radially-compact perturbations of the axial velocity component in the $\mathrm{K}-\mathrm{H}$ mode may be coherently exciting vorticity and/or entropy modes that are not modelled by PSE. The $\mathrm{K}-\mathrm{H}$ mode has more gradual radial gradients for both the radial velocity and pressure, and the corresponding fields do not display this discrepancy.

The pressure-based inner product used in the POD (see (3.1)) assigns null weights to the velocity components. Thus, for the quantitative validation of the modelled velocity fields, we define a new metric as follows

$$
\begin{aligned}
& {\left[\mathscr{A}_{T K E}\right]_{m, \omega}:=\frac{\operatorname{Re}\left\{\left\langle\hat{\boldsymbol{q}}_{m, \omega}, \hat{\boldsymbol{\Phi}}_{m, \omega}^{(1)}\right\rangle_{T K E}\right\}}{\left\|\hat{\boldsymbol{q}}_{m, \omega}\right\|_{T K E}\left\|\hat{\boldsymbol{\Phi}}_{m, \omega}^{(1)}\right\|_{T K E}},} \\
& \left\langle\hat{\boldsymbol{Q}}^{[1]}, \hat{\boldsymbol{Q}}^{[2]}\right\rangle_{T K E}:=\int_{x=0}^{20} \int_{r=0}^{5} \sum_{j \in\{x, r, \theta\}}\left\{\hat{U}_{j}^{[2]}(x, r)\right\}^{\dagger} \hat{U}_{j}^{[1]}(x, r) r \mathrm{~d} r \mathrm{~d} x .
\end{aligned}
$$

The square of the norm induced from the above inner product is proportional to the integrated incompressible turbulent kinetic energy (TKE). Only the real part of the 'alignment' is considered in the above definition since the relative phase of the PSE and POD fields is predetermined from the analysis in $\S 4$. Since the PSE solution has been scaled to agree with the first POD mode of pressure, the sole 

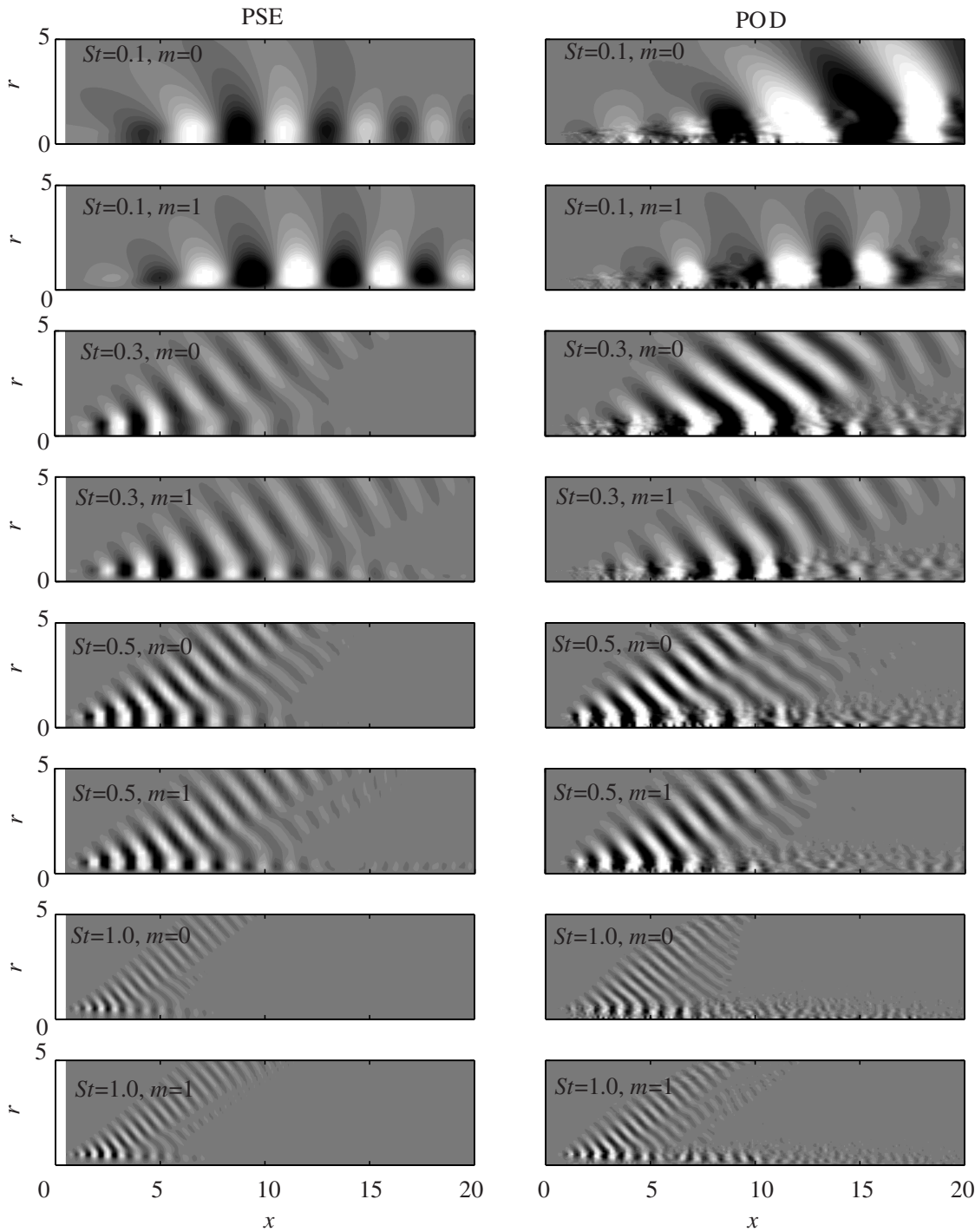

FIGURE 8. Comparison of pressure component of PSE solution with corresponding first POD modes for the heated (B122) jet. The real part of the modes are plotted with greyscale ranging within \pm 0.0043 .

meaningful comparison of the PSE velocity field is with the velocity components of this POD mode.

Figure 10 presents the alignment of the TKE in several Fourier modes for both the supersonic jets under consideration. The alignment is much poorer than that found for the pressure component, the reasons for which have been discussed above in the context of the $S t=0.5$ wavepackets. Although not shown here, the values of $\mathscr{A}_{T K E}$ were found to be more in line with $\mathscr{A}_{P}$ when the axial domain considered in the inner product of $(5.1 b)$ was restricted to $0 \leqslant x \leqslant 8$. The results in this section suggest that the linear PSE model is able to deliver encouraging predictions of the velocity fields of wavepackets educed from empirical data, but only in the region prior to their decay. 

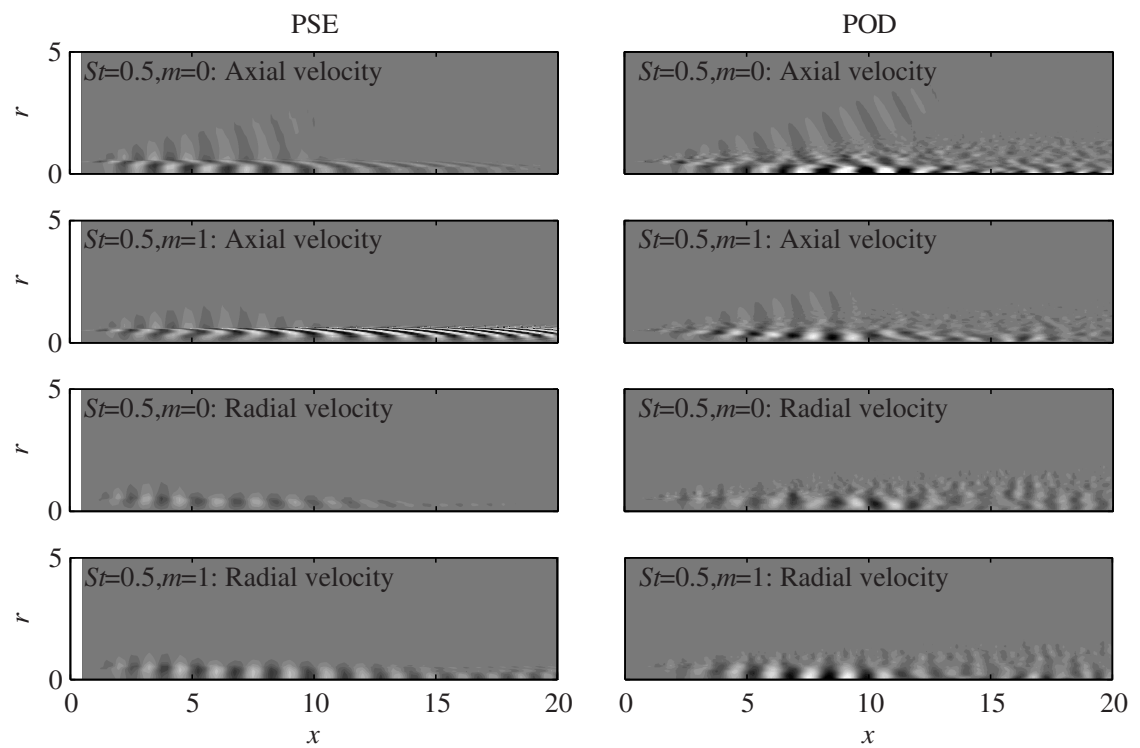

FIGURE 9. Comparison of the real parts of axial and radial velocity fields from PSE and POD for the isothermal (B118) jet, with greyscale ranging in \pm 0.025 .

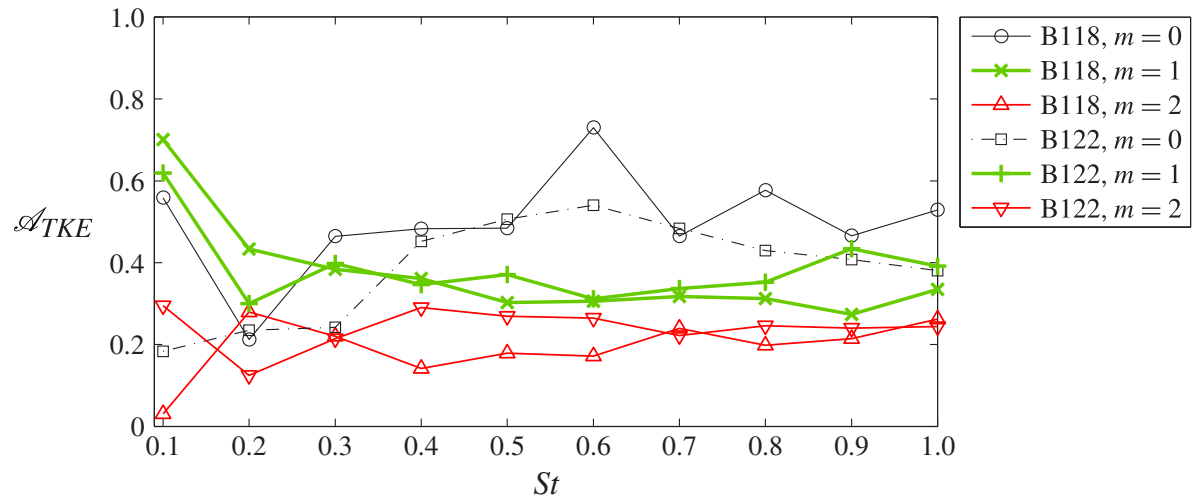

FIGURE 10. (Colour online) Alignment of the velocity components of the PSE solution with those associated with the first POD modes of pressure in the two jets.

Cavalieri et al. (2013) report on a detailed comparison of velocity fields modelled by PSE with experimental data of a Mach 0.4 jet, with similar conclusions as above.

\section{Comparisons of the acoustic field}

The formulation of the acoustic radiation problem in $\$ 2.2$ has established the need to select the radius $r_{K S}$ of the cylindrical Kirchhoff surface such that the source term $\check{\mathscr{S}}$ in (2.6) vanishes outside it but the PSE solution is valid on it. This source term is displayed for a representative PSE mode in figure 11. Following Freund (2001), $\check{\mathscr{S}}$ is computed indirectly by evaluating the left-hand side of (2.6) with the PSE pressure solution. Beyond $r=3$ the source term for this mode is at least three orders of 


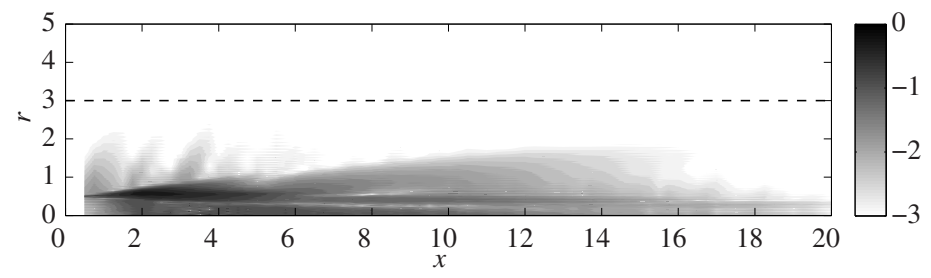

FIgURE 11. Acoustic source term for the $S t=0.4, m=0$ PSE solution in the isothermal (B118) jet, depicted by contours of $\log _{10}\left(\left|\hat{\mathscr{S}}_{m, \omega}\right|\right)$. The source is normalized to have unit maximum, and the contours are saturated below -3 .

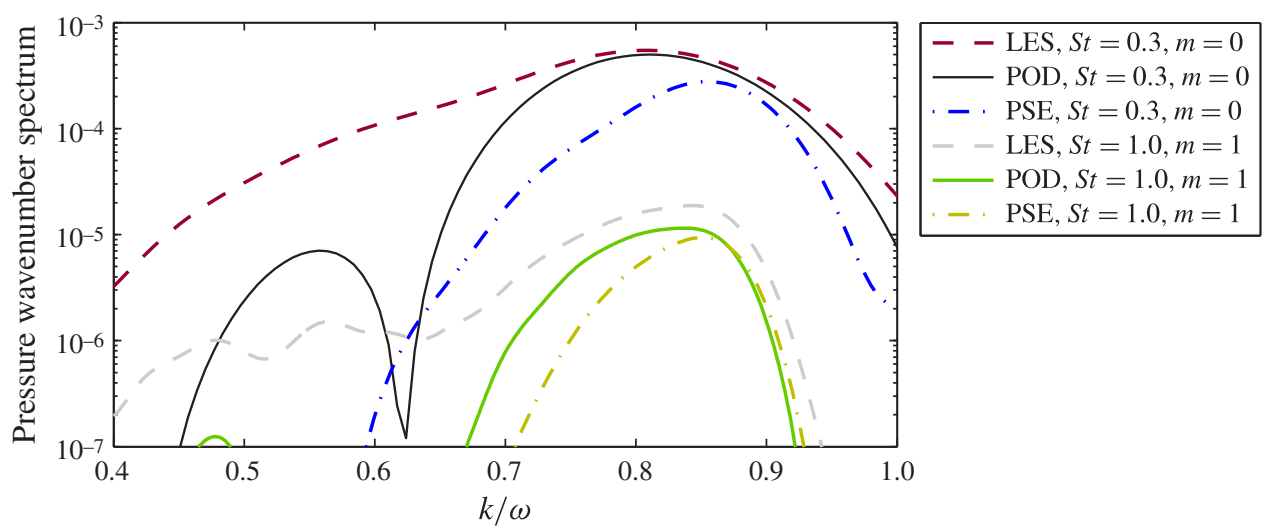

FIgURE 12. (Colour online) Axial wavenumber spectra of pressure in two Fourier modes extracted on the $r_{K S}=3$ Kirchhoff surface for the isothermal (B118) jet. Depicted are the results from the full fluctuation information in the LES database, the first POD modes of the same, as well as the corresponding scaled PSE solutions.

magnitude reduced from its maximum. Similar observations were made in all of the Fourier modes investigated in this work. The range of $r_{K S}$ available in the literature is from $1.9 D$ to $5 D$. We select a value of $3 D$ based on the evidence in figure 11 , as well as the parametric study described in appendix B; this matches the choice made by Balakumar (1998).

The axial wavenumber spectrum of pressure on the Kirchhoff surface is most relevant for the acoustic predictions (see (2.9)). The energetic portion of the radiating range of wavenumbers is depicted in figure 12 for two representative Fourier modes in the isothermal jet. Both the PSE solution and the first POD mode represent average wavepackets; their spectra agree reasonably well, as was anticipated from the match of the shape and wavelength of the contours in figure 6 .

The actual axial wavenumber spectrum of the stochastic wavepackets is obtained by ensemble-averaging the individual spectra computed from each of the $J$ segments of the LES time series (see §3.2). The resulting spectrum (termed 'LES' in figure 12) is necessarily broader than the spectrum of the corresponding first POD mode. However, since the latter extracts the most energetic coherent fluctuations, the two spectra match near their peak. Moreover, since the spectral peaks are in the radiating range, we demonstrate below that the resulting acoustic fields bear significant similarities. 
The Kirchhoff surface-based acoustic projection results for the PSE model are validated against high-fidelity predictions from the LES data using a permeable formulation of the FW-H equation (Brès et al. 2012). The latter uses the data on a conical shell with half-angle of $6.3^{\circ}$; the shell intercepts the $x=0$ plane at $r=0.6$. Although the coflow outside the FW-H surface was ignored in propagating the LES solution, the resulting far-field sound spectra demonstrated a good match with the lossless measurements at UTRC (Brès et al. 2012): an example of this has been presented in figure $2(b)$. The coflow is also ignored in propagating the pressure extracted on the Kirchhoff surface in the present work. A discrepancy is expected in the acoustic fields obtained from the FW-H and KS methods, owing to the $M_{c o}=0.1$ flow between the relevant conical and cylindrical shells. However, this error is minimized by making comparisons on a polar arc of radius $100 D$. The alternative of incorporating the coflow encounters the issue of modelling the acoustic refraction through the shear layer that arises where the coflow ceases: this detracts from the main objective of the work.

There is another source of error arising from the limited axial domain of the pressure fluctuation data extracted from the LES database for post-processing $(0 \leqslant x \leqslant 20)$. The FW-H surface, on the other hand, extended up to $x=30$ and, more importantly, the method of end caps (Shur, Spalart \& Strelets 2005) was used to account for the acoustic source flux at the downstream end of the domain (Brès et al. 2012). The consequent discrepancy is most prominent for low-frequency wavepackets that saturate far downstream, as well as for radiation to far aft angles. Thus, the comparisons in the far field are restricted to $S t \geqslant 0.3$, and polar angles less than $155^{\circ}$ (measured from the upstream jet axis). The former constraint coincides with the range of validity of the PSE solutions, as demonstrated by the near-field comparisons in $\S 4$.

The validity of (2.9) is first assessed in figure 13, which compares the acoustic spectra on the polar arc for the isothermal jet. The spectra are reported as narrowband power spectral density (PSD) in decibels per Strouhal resolution. The LES data is directly used in the FW-H and KS methods described above. Across the range of Fourier modes and the aft angles of peak radiation, the two methods are seen to yield very similar results.

Note that the acoustic results labelled 'LES: FW-H' differ from those presented by Brès et al. (2012). Here, the complex far-field pressure is predicted by the frequencydomain FW-H solver at 72 azimuthally spaced points for any $x-r$ location, and then decomposed into Fourier azimuthal modes. The resulting acoustic spectra are then binaveraged with $\Delta S t_{\text {bin }}=0.05$ (see discussion of figure 2). The 'LES: KS' spectra, on the other hand, are ensemble-averaged (see comments on figure 12). The results in figure 13 confirm the equivalence of the two averages.

Figure 13 also presents the directivity of the $r_{K S}=3$ Kirchhoff surface information extracted from the first POD modes of pressure. The directivity curves from this reduced information are seen to match the full-information results near the peak radiation angles, although there is significant under-prediction away from the peak. This result was anticipated from figure 12, which showed that the most energetic portion of the near-field pressure fluctuations (captured as an average wavepacket by the first POD mode) is well within the range of radiating wavenumbers. Thus, the intermittency of the wavepackets (which broadens the wavenumber spectrum) has relatively little contribution to the peak acoustic radiation in the isothermal supersonic jet. 


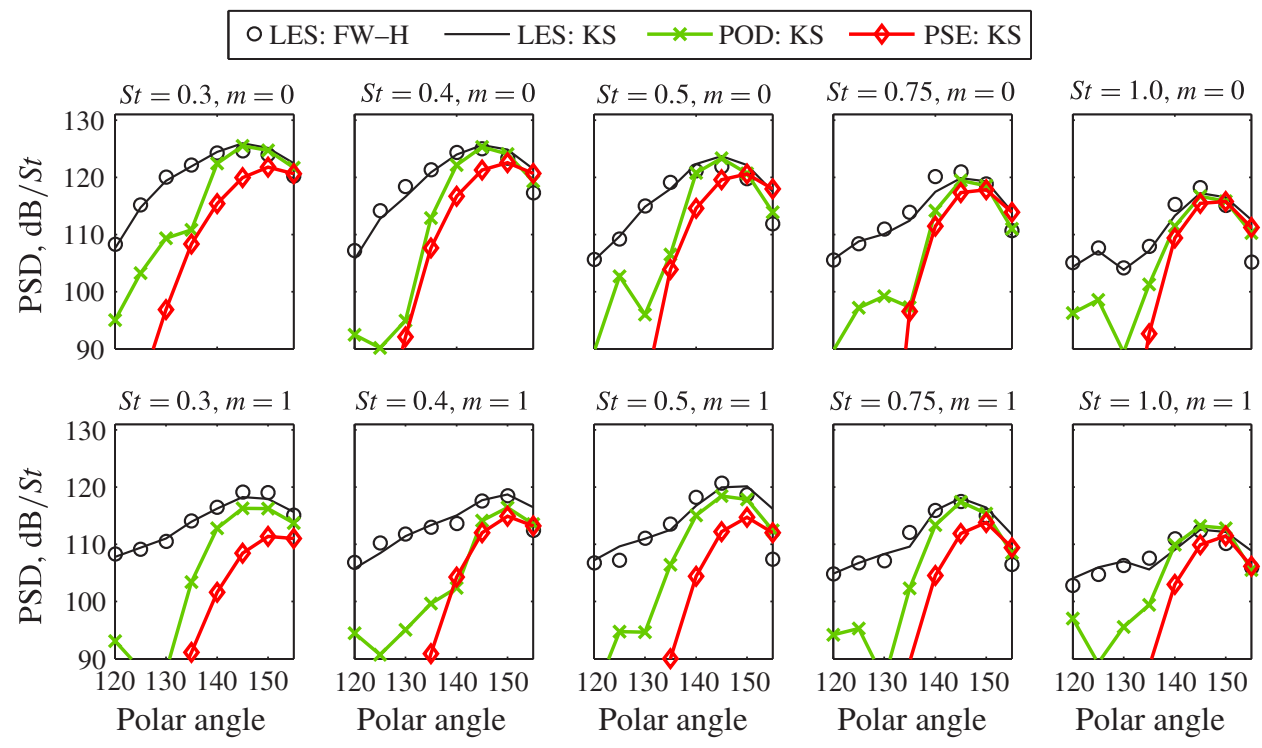

FIGURE 13. (Colour online) Directivity of far-field acoustic predictions on a polar arc of radius $100 D$ for the isothermal (B118) jet.

In contrast, in the case of a subsonic (Mach 0.9) jet, Cavalieri et al. (2011) used a different procedure to model the average wavepacket, and found it to under-predict the far-field PSD by $25 \mathrm{~dB} / S t$ at the peak radiation aft angle (see also Baqui et al. (2013) and Breakey et al. (2013)). However, agreement was much improved when the intermittency of the wavepackets was modelled empirically by these authors, as well as by Reba, Narayanan \& Colonius (2010) earlier. Thus, the role of intermittency in jet mixing noise radiation increases significantly in going from a convectively supersonic regime to a subsonic condition.

The final set of directivity curves in figure 13 are obtained by projecting the PSE solution with the KS method. The PSE modes retain their scaling from the earlier use of (4.2). For all of the Fourier modes depicted, the alignment metric had been demonstrated to be close to unity in figure 5, which explains the fairly good match between the PSE and POD directivity curves observed in figure 13. The similarities in their respective axial wavenumber spectra, exemplified in figure 12, also anticipated this result.

The encouraging agreement between the four methods for predicting the far-field directivity in the isothermal supersonic jet extends to the heated supersonic jet in figure 14. The increased acoustic Mach number of the latter causes the shift of the peak directivity to lower polar angles. This modification is replicated by the PSE model. The mismatch in the $S t=0.3, m=0$ Fourier mode was expected from the corresponding discrepancy observed in the near-field pressure in figure 8.

An interesting difference is noted between the results for the two jets. The heated jet displays superior match between the actual acoustic field and that of the average wavepacket (i.e. the first POD mode of pressure), with reduced discrepancies at off-peak polar angles. This further extends the argument made above regarding the diminishing importance of intermittency of the wavepackets in their acoustic radiation with increasing jet speed. However, the role of jet temperature in this effect cannot 


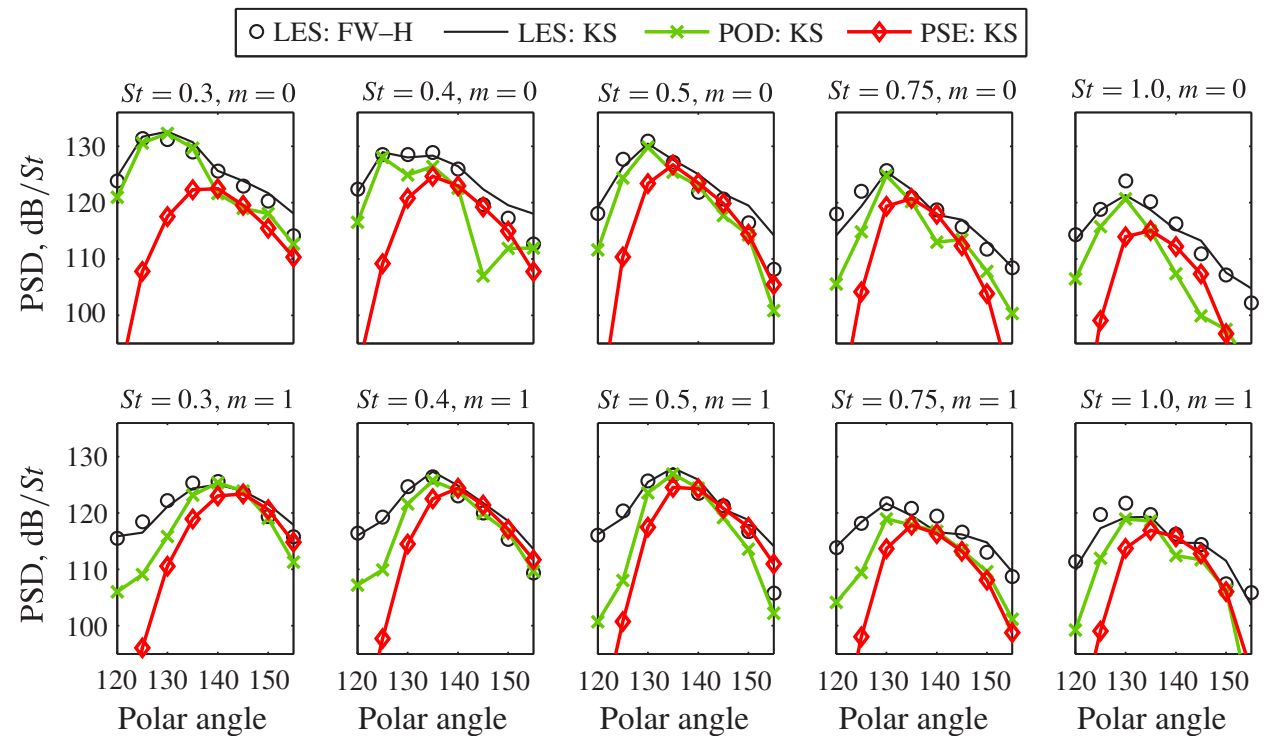

FIGURE 14. (Colour online) Directivity of far-field acoustic predictions on a polar arc of radius $100 D$ for the heated $(\mathrm{B} 122)$ jet.

be separated from that of the jet speed, since the B118 and B122 cases differ in both regards.

\section{Summary and conclusions}

The ubiquity of coherent structures in turbulent shear layers discovered in the 1960s and 1970s prompted Tam and his coworkers to propose a mechanism for supersonic jet mixing noise based on linear instability waves of the turbulent mean flow. This model was well accepted from the 1970s to the early 1990s based on qualitative agreement with the limited experimental observations then available (Tam 1995). However, as DNS databases of shear layers started appearing subsequently, the linear theory was judged to be lacking in its precise predictions (Mohseni et al. 2002; Suponitsky et al. 2010). In hindsight, we can say that these early simulations were at very low Reynolds numbers, and that their conclusions should be tempered for high-Re jets with fully turbulent shear layers (Cavalieri et al. 2013). In fact, recent works, both experimental (Zaman 2012) and computational (Bogey, Marsden \& Bailly 2012), demonstrate the critical role that the boundary-layer state at the nozzle exit plays in jet noise. The highly disturbed but nominally laminar boundary layer states at the exit of low-Re jets are associated with higher noise than the turbulent conditions in high-Re cases, which provides support for the earlier linear theory of turbulent jet noise generation.

Recently, Gudmundsson \& Colonius (2011) reported a model based on PSE which delivered excellent agreement with detailed experimental data in the near field of turbulent subsonic jets (see also Jordan \& Colonius (2013) and Cavalieri et al. (2013)). The comparisons were materially improved on first educing the low-energy but spatially coherent wavepackets by applying a spatiotemporal filter based on POD to the data. Continuing on their success, we herein revisit the supersonic jet noise problem with the PSE model, and quantitatively demonstrate that Tam's 
original insight was indeed accurate. Towards this, we report rigourous and extensive validations against a high-fidelity LES database of two ideally expanded Mach 1.5 jets, one isothermal and the other moderately heated (Brès et al. 2012).

This article makes two main contributions. It demonstrates the validity of linear instability wave models for predicting the average wavepacket evolution in supersonic jets. The pressure and velocity fields associated with the wavepackets are modelled with reasonable accuracy in their growing regime. The agreement is much poorer in the decay zone within the jet shear layer, although the pressure in the irrotational near field continues to display a fair match. The PSE model is initiated with the $\mathrm{K}-\mathrm{H}$ mode of the locally parallel linear stability problem at a cross-section near the nozzle, and the downstream march of the solution tracks this mode. Unlike subsonic jets, the supersonic jet supports two other families of unstable modes (Tam \& $\mathrm{Hu}$ 1989). However, since the K-H mode undergoes the largest amplification, it governs the wavepacket dynamics (Rodríguez et al. 2013) and, consequently, sound radiation. As argued by Gudmundsson \& Colonius (2011) for the subsonic case, although the wavepackets appear to be linear perturbations of the turbulent mean flow on average, this does not rule out the importance of nonlinear interactions in establishing the turbulence cascade in an instantaneous sense.

The second contribution of this article is in concurrently predicting the salient features of the acoustic field. This not only reflects our success in modelling the average wavepackets, but also the relative unimportance of the unsteadiness or intermittency (i.e. second-order statistics) of these convectively supersonic wavepackets in determining the acoustic field. The latter is a consequence of the energetic wavenumbers of the near-field pressure being in the radiating range. In contrast, the energy of convectively subsonic wavepackets is primarily in non-radiating wavenumbers, so that intermittency assumes a dominant role in determining their acoustic field.

\section{Acknowledgements}

The authors gratefully acknowledge support from the Office of Naval Research under contract N0014-11-1-0753 with Dr B. Henderson as technical monitor, and from NAVAIR under STTR contract N68335-11-C-0026 managed by Dr J. Spyropoulos. Any opinions, findings and conclusions or recommendations expressed in this material are those of the authors and do not necessarily reflect the views of the sponsoring agencies. The majority of the large eddy simulations were conducted on CRAY XE6 machines at DoD supercomputer facilities in ERDC and AFRL. D.R. acknowledges funding from the European Union Marie Curie - COFUND program. Finally, the authors thank the anonymous reviewing panel for many insightful suggestions.

\section{Appendix A. Governing equations}

We list below the set of linearized equations governing the jets under consideration:

$$
\begin{gathered}
\frac{\partial \boldsymbol{u}^{\prime}}{\partial t}+\overline{\boldsymbol{u}} \cdot \nabla \boldsymbol{u}^{\prime}+\boldsymbol{u}^{\prime} \cdot \nabla \overline{\boldsymbol{u}}+\bar{\zeta} \nabla p^{\prime}-\frac{M_{a}}{\operatorname{Re}}\left\{\bar{\zeta}\left(\nabla^{2} \boldsymbol{u}^{\prime}+1 / 3 \nabla \mathscr{D}^{\prime}\right)\right. \\
\left.+\zeta^{\prime}\left(\nabla^{2} \overline{\boldsymbol{u}}+1 / 3 \nabla \overline{\mathscr{D}}\right)\right\}=C_{u}, \\
\frac{\partial p^{\prime}}{\partial t}+\overline{\boldsymbol{u}} \cdot \nabla p^{\prime}+\mathscr{D}^{\prime}+\gamma \overline{\mathscr{D}} p^{\prime}-\frac{\gamma M_{a}}{\operatorname{RePr}}\left(\bar{\zeta} \nabla^{2} p^{\prime}+\frac{1}{\gamma} \nabla^{2} \zeta^{\prime}+p^{\prime} \nabla^{2} \bar{\zeta}\right) \\
-\frac{(\gamma-1) M_{a}}{\operatorname{Re}}\left[\left\{(\nabla \overline{\boldsymbol{u}})+(\nabla \overline{\boldsymbol{u}})^{\mathrm{T}}\right\}:\left\{\left(\nabla \boldsymbol{u}^{\prime}\right)+\left(\nabla \boldsymbol{u}^{\prime}\right)^{\mathrm{T}}\right\}-4 / 3 \overline{\mathscr{D}} \mathscr{D}^{\prime}\right]=C_{p},
\end{gathered}
$$



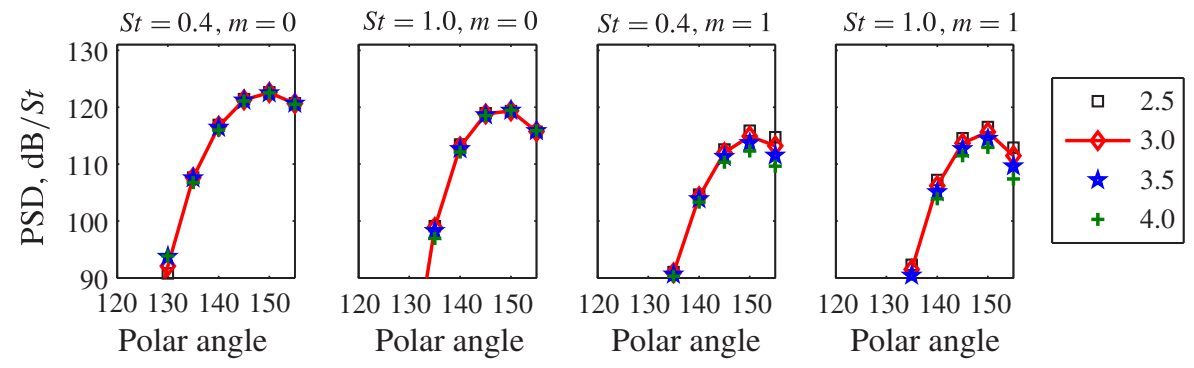

FIGURE 15. (Colour online) Far-field acoustic predictions from PSE on a polar arc of radius $100 D$ for the isothermal (B118) jet with various choices of the cylindrical Kirchhoff surface radius, $r_{K S}$.

$$
\frac{\partial \zeta^{\prime}}{\partial t}+\overline{\boldsymbol{u}} \cdot \nabla \zeta^{\prime}+\boldsymbol{u}^{\prime} \cdot \nabla \bar{\zeta}-\zeta^{\prime} \overline{\mathscr{D}}-\bar{\zeta} \mathscr{D}^{\prime}=C_{\zeta} .
$$

Here, $\mathscr{D}$ denotes the dilatation. The symbols $C_{u}, C_{p}$ and $C_{\zeta}$ denote constant terms arising since the base flow does not satisfy the governing equations; these terms do not affect the linearized dynamics at the non-zero frequencies that are of interest here. Specific volume is preferred over density in the formulation as the nonlinearity in the original governing equations is rendered quadratic instead of cubic (Iollo, Lanteri \& Desideri 2000); this also serves to simplify the linearized equations above.

\section{Appendix B. Choice of the Kirchhoff surface location}

As long as the Kirchhoff surface is located in the linear acoustic field of the jet, the far field projected from it should be independent of the precise choice of its radius, $r_{K S}$. Four different values of $r_{K S}$ are tested in figure 15 for the B118 jet; the metric is the directivity diagram (of the kind discussed in $\S 6$ ) for four representative Fourier modes. The results for the $m=0$ modes are well converged. For the $m=1$ modes, the spectral levels display a weak dependence on the choice of $r_{K S}$, which reflects the small errors incurred by PSE in modelling the near-field acoustics.

\section{REFERENCES}

Alkislar, M. B., Krothapalli, A. \& Butler, G. W. 2007 The effect of streamwise vortices on the aeroacoustics of a Mach 0.9 jet. J. Fluid Mech. 578, 139-169.

BAlakumar, P. 1998 Prediction of supersonic jet noises. In 36th AIAA Aerospace Sciences Meeting, AIAA Paper 1998-1057.

Baqui, Y., Agarwal, A., Cavalieri, A. V. G. \& Sinayoko, S. 2013 Nonlinear and linear noise source mechanisms in subsonic jets. In 19th AIAA/CEAS Aeroacoustics Conference, AIAA Paper 2013-2087.

Bogey, C., Marsden, O. \& Bailly, C. 2012 Influence of initial turbulence level on the flow and sound fields of a subsonic jet at a diameter-based Reynolds number of $10^{5}$. J. Fluid Mech. 701, 352-385.

Breakey, D. E. S., Jordan, P., Cavalieri, A. V. G., Léon, O., Zhang, M., Lehnasch, G., Colonius, T. \& Rodríguez, D. 2013 Near-field wavepackets and the far-field sound of a subsonic jet. In 19th AIAA/CEAS Aeroacoustics Conference, AIAA Paper 2013-2083.

Brès, G. A., Nichols, J. W., Lele, S. K. \& HAM, F. E. 2012 Towards best practices for jet noise predictions with unstructured large eddy simulations. In 42nd AIAA Aerospace Sciences Meeting and Exhibit, AIAA Paper 2012-2965. 
Brown, G. L. \& Roshko, A. 1974 On density effects and large structure in turbulent mixing layers. J. Fluid Mech. 64 (4), 775-816.

Cavalieri, A. V. G., Jordan, P., Agarwal, A. \& Gervais, Y. 2011 Jittering wave-packet models for subsonic jet noise. J. Sound Vib. 330 (18-19), 4474-4492.

Cavalieri, A. V. G., Rodríguez, D., Jordan, P., Colonius, T. \& Gervais, Y. 2013 Wavepackets in the velocity field of turbulent jets. J. Fluid Mech. 730, 559-592.

Cheung, L. C. \& Lele, S. K. 2009 Linear and nonlinear processes in two-dimensional mixing layer dynamics and sound radiation. J. Fluid Mech. 625, 321-351.

Crighton, D. G. \& Gaster, M. 1976 Stability of slowly diverging jet flow. J. Fluid Mech. 77 (2), 397-413.

Crow, S. \& Champagne, F. 1971 Orderly structure in jet turbulence. J. Fluid Mech. 48 (3), $547-591$.

Day, M. J., Mansour, N. N. \& Reynolds, W. C. 2001 Nonlinear stability and structure of compressible reacting mixing layers. J. Fluid Mech. 446, 375-408.

FREUND, J. B. 1997 Compressibility effects in a turbulent axisymmetric mixing layer. PhD thesis, Stanford University.

Freund, J. B. 2001 Noise sources in a low-Reynolds-number turbulent jet at Mach 0.9. J. Fluid Mech. 438 (1), 277-305.

Freund, J. B. \& Colonius, T. 2009 Turbulence and sound-field POD analysis of a turbulent jet. Intl J. Aeroacoust. 8 (4), 337-354.

Goldstein, M. E. \& Leib, S. J. 2005 The role of instability waves in predicting jet noise. J. Fluid Mech. 525, 37-72.

Gudmundsson, K. \& Colonius, T. 2011 Instability wave models for the near-field fluctuations of turbulent jets. J. Fluid Mech. 689, 97-128.

Henderson, B. 2010 Fifty years of fluidic injection for jet noise reduction. Intl J. Aeroacoust. 9 (1-2), 91-122.

Herbert, T. 1997 Parabolized stability equations. Annu. Rev. Fluid Mech. 29, 245-283.

IOllo, A., LANTERI, S. \& DESIDERI, J.-A. 2000 Stability properties of POD-Galerkin approximations for the compressible Navier-Stokes equations. Theor. Comput. Fluid Dyn. 13 (6), 377-396.

Jordan, P. \& Colonius, T. 2013 Wave packets and turbulent jet noise. Annu. Rev. Fluid Mech. 45, 173-195.

Kerhervé, F., Jordan, P., Cavalieri, A. V. G., Delville, J., Bogey, C. \& Juvé, D. 2012 Educing the source mechanism associated with downstream radiation in subsonic jets. J. Fluid Mech. 710, 606-640.

LI, F. \& Malik, M. R. 1997 Spectral analysis of parabolized stability equations. Comput. Fluids 26 (3), 279-297.

Lighthill, M. J. 1952 On sound generated aerodynamically. I. General theory. Proc. R. Soc. Lond.A 211 (1107), 564-587.

Lin, R.-S., Reba, R., Narayanan, S., Hariharan, N. S. \& Bertolotti, F. P. 2004 Parabolized stability equation based analysis of noise from an axisymmetric hot jet. In Proceedings of ASME FEDSM, HT-FED2004-56820.

Malkus, W. V. R. 1956 Outline of a theory of turbulent shear flow. J. Fluid Mech. 1 (5), 521-539.

MANKBADI, R. \& LIU, J. T. C. 1984 Sound generated aerodynamically revisited: large-scale structures in a turbulent jet as a source of sound. Proc. R. Soc. Lond. A 311 (1516), 183-217.

Mattingly, G. E. \& Chang, C. C. 1974 Unstable waves on an axisymmetric jet column. J. Fluid Mech. 65 (3), 541-560.

Michalke, A. 1984 Survey on jet instability theory. Prog. Aerosp. Sci. 21, 159-199.

Mohseni, K. \& Colonius, T. 2000 Numerical treatment of polar coordinate singularities. J. Comput. Phys. 157, 787-795.

Mohseni, K., Colonius, T. \& Freund, J. B. 2002 An evaluation of linear instability waves as sources of sound in a supersonic turbulent jet. Phys. Fluids 14 (10), 3593-3600.

Mollo-Christensen, E. 1963 Measurements of near field pressure of subsonic jets. Tech. Rep. NATO A.G.A.R.D. Report 449.

Mollo-Christensen, E. 1967 Jet noise and shear flow instability seen from an experimenter's point of view. J. Appl. Mech. 34 (1), 1-7. 
Morse, P. M. \& Ingard, K. 1968 Theoretical Acoustics. McGraw-Hill.

Piot, E., Casalis, G., Muller, F. \& Bailly, C. 2006 Investigation of the PSE approach for subsonic and supersonic hot jets. Detailed comparisons with LES and linearized Euler equations results. Intl J. Aeroacoust. 5 (4), 361-393.

Reba, R., Narayanan, S. \& Colonius, T. 2010 Wave-packet models for large-scale mixing noise. Intl J. Aeroacoust. 9 (4-5), 533-558.

Rodríguez, D., Sinha, A., Brès, G. \& Colonius, T. 2013 Inlet conditions for wave packet models in turbulent jets based on eigenmode decomposition of large eddy simulation data. Phys. Fluids 25, 105107.

Samimy, M., Kim, J.-H., Kearney-Fischer, M. \& Sinha, A. 2012 High-speed and high Reynolds number jet control using localized arc filament plasma actuators. J. Propul. Power 28 (2), 269-280.

Schlinker, R. H., Simonich, J. C., Shannon, D. W., Reba, R., Colonius, T., Gudmundsson, K. \& LADIENDE, F. 2009 Supersonic jet noise from round and chevron nozzles: experimental studies. In 15th AIAA/CEAS Aeroacoustics Conference, AIAA Paper 2009-3257.

Shur, M. L., Spalart, P. R. \& Strelets, M. K. 2005 Noise prediction for increasingly complex jets. Part I: methods and tests. Intl J. Aeroacoust. 4 (3-4), 213-246.

Sirovich, L. 1987 Turbulence and the dynamics of coherent structures, Parts I-III. Q. Appl. Maths XLV (3), 561-590.

Suponitsky, V., SANDham, N. D. \& Morfey, C. L. 2010 Linear and nonlinear mechanisms of sound radiation by instability waves in subsonic jets. J. Fluid Mech. 658, 509-538.

Suzuki, T. \& Colonius, T. 2006 Instability waves in a subsonic round jet detected using a near-field phased microphone array. J. Fluid Mech. 565, 197-226.

TAM, C. K. W. 1971 Directional acoustic radiation from a supersonic jet generated by shear layer instability. J. Fluid Mech. 46 (4), 757-768.

TAM, C. K. W. 1991 Jet noise generated by large-scale coherent motion. In Aeroacoustics of flight vehicles: Theory and practice. Volume 1: Noise sources (ed. H. H. Hubbard), pp. 311-390. NASA RP-1258.

TAM, C. K. W. 1995 Supersonic jet noise. Annu. Rev. Fluid Mech. 27 (1), 17-43.

TAM, C. K. W. \& BURTON, D. E. 1984 Sound generated by instability waves of supersonic flows. Part 2. Axisymmetric jets. J. Fluid Mech. 138, 273-295.

TAM, C. K. W. \& ChEn, P. 1994 Turbulent mixing noise from supersonic jets. AIAA J. 32 (9), $1774-1780$.

TAM, C. K. W. \& HU, F. Q. 1989 On the three families of instability waves of high-speed jets. J. Fluid Mech. 201, 447-483.

TAM, C. K. W. \& Morris, P. J. 1985 Tone excited jets, Part V: a theoretical model and comparison with experiment. J. Sound Vib. 102, 119-151.

Thompson, K. W. 1987 Time dependent boundary conditions for hyperbolic systems. J. Comput. Phys. 68, 1-24.

Troutt, T. R. \& Mclaughlin, D. K. 1982 Experiments on the flow and acoustic properties of a moderate-Reynolds-number supersonic jet. J. Fluid Mech. 116, 123-156.

Vreman, A. W. 2004 An eddy-viscosity subgrid-scale model for turbulent shear flow: algebraic theory and applications. Phys. Fluids 16 (10), 3670-3681.

Yen, C. C. \& Messersmith, N. L. 1998 Application of parabolized stability equations to the prediction of jet instabilities. AIAA J. 36 (8), 1541-1544.

Yen, C. C. \& Messersmith, N. L. 1999 The use of compressible parabolized stability equations for prediction of jet instabilities and noise. In 5th AIAA/CEAS Aeroacoustics Conference, AIAA Paper 1999-1859.

You, D. \& MoIN, P. 2007 A dynamic global-coefficient subgrid-scale eddy-viscosity model for large-eddy simulation in complex geometries. Phys. Fluids 19, 065110.

ZamAn, K. B. M. Q. 2012 Effect of initial boundary-layer state on subsonic jet noise. AIAA J. 50 (8), 1784-1795. 